ARTICLE

\title{
A magma ocean origin to divergent redox evolutions of rocky planetary bodies and early atmospheres
}

\author{
Jie Deng (iD ${ }^{1,4 凶}$, Zhixue Du (iD) ${ }^{2 凶}$, Bijaya B. Karki ${ }^{3}$, Dipta B. Ghosh ${ }^{3}$ \& Kanani K. M. Lee (iD ${ }^{1,5}$
}

\begin{abstract}
Magma oceans were once ubiquitous in the early solar system, setting up the initial conditions for different evolutionary paths of planetary bodies. In particular, the redox conditions of magma oceans may have profound influence on the redox state of subsequently formed mantles and the overlying atmospheres. The relevant redox buffering reactions, however, remain poorly constrained. Using first-principles simulations combined with thermodynamic modeling, we show that magma oceans of Earth, Mars, and the Moon are likely characterized with a vertical gradient in oxygen fugacity with deeper magma oceans invoking more oxidizing surface conditions. This redox zonation may be the major cause for the Earth's upper mantle being more oxidized than Mars' and the Moon's. These contrasting redox profiles also suggest that Earth's early atmosphere was dominated by $\mathrm{CO}_{2}$ and $\mathrm{H}_{2} \mathrm{O}$, in contrast to those enriched in $\mathrm{H}_{2} \mathrm{O}$ and $\mathrm{H}_{2}$ for Mars, and $\mathrm{H}_{2}$ and $\mathrm{CO}$ for the Moon.
\end{abstract}

\footnotetext{
${ }^{1}$ Department of Geology and Geophysics, Yale University, New Haven, CT 06511, USA. ${ }^{2}$ State key Laboratory of Isotope Geochemistry, Guangzhou Institute of Geochemistry, Chinese Academy of Sciences, 510640 Guangzhou, China. ${ }^{3}$ School of Electrical Engineering and Computer Science, Department of Geology and Geophysics, and Center for Computation and Technology, Louisiana State University, Baton Rouge, LA 70803, USA. ${ }^{4}$ Present address: Earth, Planetary, and Space Sciences, University of California, Los Angeles, CA 90095, USA. ${ }^{5}$ Present address: Lawrence Livermore National Laboratory, Livermore,

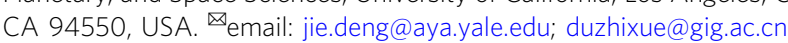


T he redox condition of planetary bodies influences their chemical differentiation and governs the composition of overlying atmospheres ${ }^{1-5}$. For instance, to understand how bio-essential volatiles such as carbon and hydrogen were initially incorporated near Earth's surface requires knowledge about the redox state during early stages of Earth's history. A number of studies have shown that the uppermost mantle of present-day Earth is considerably oxidized (IW + 3.5, that is, $3.5 \log$ units above the iron-wüstite buffer) ${ }^{6}$. Petrological evidence also suggests that such oxidized conditions formed early, 4.3-4.4 Ga ago ${ }^{7}$. Unlike Earth, the present-day Martian and Lunar mantles are considered to be much more reduced $(\sim \mathrm{IW}-1)^{8-11}$.

These contrasting oxidization states may have been set up during the early phase of planetary formation when magma oceans (MOs) could have existed ${ }^{3,12}$. Such an MO involved mechanism has yet to be fully established because relevant redox controlling reactions are still poorly constrained in realistic magma ocean scenarios. Several studies have inferred the oxygen fugacity $\left(f_{\mathrm{O}_{2}}\right)$ profile of silicate melts at high pressures using the experimental data at zero or relatively low pressures and is applicable only for shallow magma oceans ${ }^{13,14}$. The oxygen fugacity is a function of pressure, temperature, and composition, thus likely varying greatly within MOs that could have extended very deep, even covering the entire mantle regime.

Here, we study the redox controlling reactions in magma oceans by simulating silicate melts containing ferrous and ferric iron with first-principles molecular dynamics (FPMD) and perform thermodynamic modeling at pressures that cover the entire Earth's mantle and temperatures up to $5000 \mathrm{~K}$. The results suggest that ferric iron becomes increasingly energetically favorable with pressure mainly due to its small partial molar volume in silicate melts under large compression. Consequently, the magma oceans of Earth, Mars, and the Moon, if compositionally homogeneous due to vigorous mixing, would be characterized with a vertical gradient in oxygen fugacity. Specifically, a deeper magma ocean existing in the early Earth would have more oxidizing surface conditions compared with those of smaller bodies like Mars and the Moon. The contrasting surface conditions between these planetary bodies suggest that the early atmosphere in equilibrium with Earth's surface may have been dominated by $\mathrm{CO}_{2}$ and $\mathrm{H}_{2} \mathrm{O}$, in contrast to those enriched in $\mathrm{H}_{2} \mathrm{O}$ and $\mathrm{H}_{2}$ for Mars and $\mathrm{H}_{2}$ and $\mathrm{CO}$ for the Moon.

\section{Results and discussion}

Equations of state of silicate melts. At the base of a MO where metallic melts may pond before sinking into the cor $\mathrm{e}^{15}$, the oxygen fugacity is governed by the equilibrium between the metallic and silicate melts, and can be directly calculated given the compositions of these melts are known. Away from the base where metallicmeltis absent due to its rapid sinking velocity ${ }^{16}$, the MO redox state is controlled by the following redox buffering reaction ${ }^{3,17}$ :

$$
\mathrm{FeO}(\text { melt })+\frac{1}{4} \mathrm{O}_{2}=\mathrm{FeO}_{1.5} \text { (melt) }
$$

The thermodynamic behavior of the above reaction informs how oxygen fugacity varies with temperature and pressure. Taking the oxygen fugacity at the MO base as the boundary condition, one may, in principle, obtain the oxygen fugacity throughout the MO if the thermodynamic properties of the reactants and products in Eq. 1 are known. One key-parameter is the difference in molar volumes between $\mathrm{FeO}_{1.5}$ and $\mathrm{FeO}$ in the melts, $\Delta V$. Its value has been directly measured only at 1 bar $^{18}$ and also inferred from experiments performed up to $23 \mathrm{GPa}$ and $\sim 2500 \mathrm{~K}^{12,13,18-23}$. However, these conditions are still far from what are expected in MOs of Earth and Mars. Our goal is to calculate $\Delta V$ as a function of pressure, temperature, and composition so that we can constrain oxygen fugacity in the redox buffering Eq. (1) under directly applicable conditions. Moreover, we evaluate the MO redox states of Earth, Mars, and the Moon in order to understand their oxidation conditions of the present-day mantle and the chemistry of earliest atmosphere.

We first present the results from FPMD simulations of ironbearing $\mathrm{MgSiO}_{3}$ liquids with iron in different valence states at $2000-4000 \mathrm{~K}$ and up to $140 \mathrm{GPa}$ (Methods). The calculated pressure-volume-temperature $(P-V-T)$ relationships can be described with the following equation:

$$
P(V, T)=P\left(V, T_{0}\right)+B_{T H}\left(T-T_{0}\right)
$$

Here $P\left(V, T_{0}\right)$ represents the reference isotherm at $T_{0}=$ $3000 \mathrm{~K}$ using a fourth-order Birch-Murnaghan equation of state. The second term contains a thermal pressure coefficient, $B_{\mathrm{TH}}(V)=\left[a-b\left(\frac{V}{V_{0}}\right)+c\left(\frac{V}{V_{0}}\right)^{2}\right] / 1000$ where $a, b$, and $c$ are constants for a given melt composition. The bulk moduli of the $\mathrm{Fe}^{2+}$-bearing melts are systematically larger than those of the $\mathrm{Fe}^{3+}$ bearing melts (Supplementary Table 1). This means that the $\mathrm{Fe}^{3+}$ bearing melts are more compressible at the conditions investigated (Fig. 1), consistent with previous low-pressure studies $^{18,24}$.

Using the pressure-volume results of simulated silicate melts for the same molar content of $\mathrm{Fe}^{3+}$ and $\mathrm{Fe}^{2+}$, we calculate the difference in molar volume $(\Delta V)$ between $\mathrm{FeO}_{1.5}$ and $\mathrm{FeO}$ in the melts as a function of pressure (Fig. 1). Our calculated value of $\Delta V$ at zero pressure agrees well with existing experimental data $^{18,24}$ (Supplementary Fig. 1). As pressure increases, $\Delta V$ decreases rapidly initially in the low-pressure regime. Thereafter, $\Delta V$ increases slightly and then decreases gradually at higher pressures. The predicted non-monotonicpressure trend weakens at higher temperatures. For silicate melts of different iron contents (i.e., 12.5 and $25 \mathrm{~mol} \%), \Delta V$ takes slightly different values, showing a weak positive trend with iron content. This is consistent with the observed weak dependency of $\Delta V$ on the melt composition ${ }^{18}$. Our results thus show that $\Delta V$ remains positive at all pressures up to $140 \mathrm{GPa}$ irrespective of temperature and composition. This finding contradicts previous inferences that $\Delta V$ would keep on decreasing and eventually become negative within the pressure range of Earth's mantle 3,12 .

Previous models on $\Delta V$ either adopt a bulk modulus derivative of 4 or use an equation of state fit to experimental data within a limited pressure range ${ }^{12-14,23}$. We compare model values with our calculated results for silicate melts of $12.5 \mathrm{~mol} \%$ iron, as these models are designed for Earth's relevant composition (Supplementary Fig. 1). At low pressures $(<10 \mathrm{GPa})$, our results are in good agreement with the recent model by ref. ${ }^{12}$, both showing a sharp decrease of $\Delta V$ at low pressures, whereas at higher pressures, our results are in better line with other earlier models ${ }^{13,14,23}$, all showing that $\Delta V$ gradually levels out. These $\Delta V$ differences arise mainly due to the different pressure dependencies of the incompressibility $\left(K^{\prime}\right)$ of $\mathrm{FeO}_{1.5}$ and $\mathrm{FeO}$ in silicate melts adopted by the previous studies. Our 4th order Birch-Murnaghan fit yields a lightly larger $K^{\prime}$ for $\mathrm{FeO}_{1.5}$ (4.6) than that of $\mathrm{FeO}$ (3.3). Previous studies other than ref. ${ }^{12}$ assume $K^{\prime}$ of $\mathrm{FeO}_{1.5}$ and $\mathrm{FeO}$ to be 4, thus exhibiting similar pressure dependency of $\Delta V$ to our study. The contrasting behavior of $\Delta V$ from ref. ${ }^{12}$ is caused by drastically different $K^{\prime}$ values, 1.3 and 8 , respectively, for $\mathrm{FeO}_{1.5}$ and $\mathrm{FeO}$. These extreme values of $K^{\prime}$ are not consistent with other experimental studies on silicate melts for which $K^{\prime}$ is $3-8^{25,26}$ and on FeO liquid for which $K^{\prime}$ is $3-4^{27,28}$. The reason for this inconsistency is, however, unclear. Our analysis of the coordination environment of iron in the 

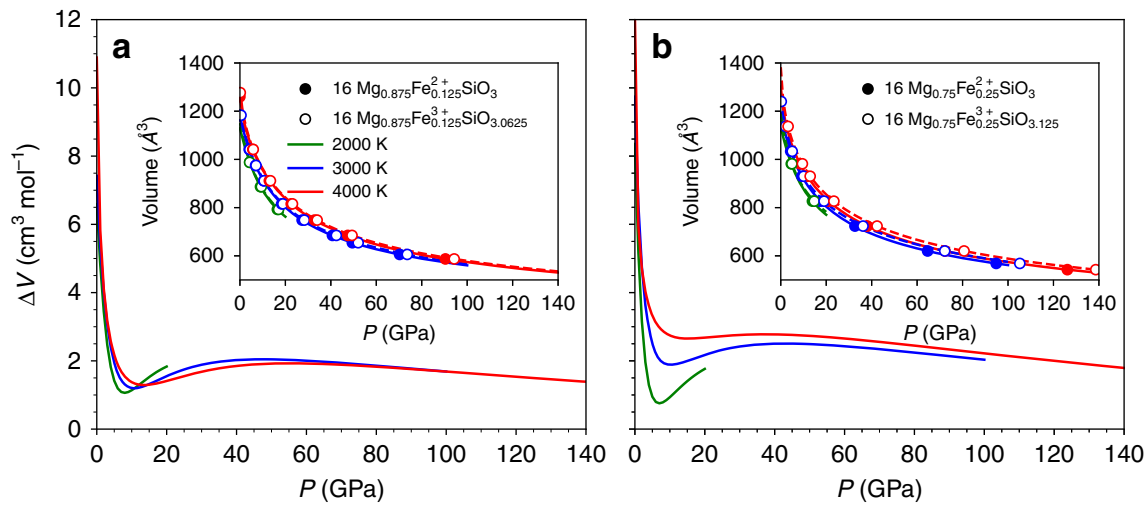

Fig. 1 Molar volume difference $(\Delta \mathbf{V})$ between $\mathbf{F e O}_{\mathbf{1 . 5}}$ and $\mathbf{F e O}$ in silicate melts. The calculated $\Delta V$ is shown as a function of pressure at different temperatures: a $12.5 \mathrm{~mol} \%$ iron for Earth- and Moon-like magma ocean and $\mathbf{b} 25 \mathrm{~mol} \%$ iron for Mars-like magma ocean. Insets show the corresponding pressure-volume relationships for melts containing 12.5 and $25 \mathrm{~mol} \%$ iron as $\mathrm{Fe}^{2+}$ (solid symbols and curves) and $\mathrm{Fe}^{3+}$ (open symbols and dashed curves). Volumes are plotted along isotherms only to pressures where the simulated systems were in a liquid state. The $1 \sigma$ standard deviation of $\Delta V$ is $\sim 0.2-0.5 \mathrm{~cm}^{3} \mathrm{~mol}^{-1}$.

silicate melts shows that the mean Fe-O coordination increases rapidly initially with pressure and more gradually at pressures beyond $40 \mathrm{GPa}$ (Supplementary Fig. 2). This pressure trend is similar to that of $\Delta V$. This implies an inherent correlation between the local iron-oxygen bonding environment in the silicate melt and $\Delta V$.

We stress that our first-principles results make no assumption on the value of $K^{\prime}$ of $\mathrm{FeO}_{1.5}$ and $\mathrm{FeO}$, so they are directly applicable over the entire mantle regime of Earth. To explore this implication further, we evaluate $\Delta V$ along two representative magma ocean thermal profiles referred to as "cold" and "hot" hereafter (Supplementary Fig. 3). The calculated $\Delta V$ varies considerably but remains positive over wide ranges of pressure and temperature of magma ocean relevance (Supplementary Fig. 4), thus indicating a positive contribution of pressure to $f_{\mathrm{O}_{2}}$.

Redox profiles. Our calculated $\Delta V$ profiles along the magma ocean thermal profiles are used to assess the redox state of magma oceans of relevance to Earth, Mars, and the Moon. We assume that the MOs are fully convective and well-mixed, resulting in a homogeneous $\mathrm{Fe}^{3+}$ to the total $\mathrm{Fe}$ ratio $\left(\mathrm{Fe}^{3+} / \Sigma \mathrm{Fe}\right)^{3}$. The thermodynamic relationship for the reaction (1) is

$$
\begin{array}{r}
-\frac{\Delta G_{r}^{0}\left(P_{0}, T\right)+\int_{P_{0}}^{P} \Delta V(P, T) d P}{R T}=\ln \frac{X_{\mathrm{FeO}}^{\text {melt }}}{X_{\mathrm{FeO}}^{\text {melt }}} \\
+\ln \frac{\gamma_{\mathrm{FeO}_{1.5}^{\text {melt }}}^{\gamma_{\mathrm{FeO}}^{\text {melt }}}}{R T}-\frac{1}{4} \ln f_{\mathrm{O}_{2}},
\end{array}
$$

where $\Delta G_{r}^{0}\left(P_{0}, T\right)$ is the free energy of the reaction (Eq.(1)) at reference pressure $P_{0}(1 \mathrm{bar})$ and temperature $T, X$ and $\gamma$ are the molar fractions and activity coefficients of the Fe-oxide component, respectively, $f_{\mathrm{O}_{2}}$ is the oxygen fugacity, and $R$ is the gas constant. The above equation has been widely used in many literatures ${ }^{3,12,13,23}$ and it suggests that the variation of $f_{\mathrm{O}_{2}}$ with pressure explicitly hinges on $\Delta V$ only. However, one should note that $\Delta V(P, T)$ not only depends on pressure and temperature but also implicitly on many extensive properties, including the configuration entropy, and excess enthalpy. We first evaluate $\Delta G_{r}^{0}\left(P_{0}, T\right)$ for $\mathrm{FeO}_{1.5}, \mathrm{FeO}$, and $\mathrm{O}_{2}$ as a function of temperature (Supplementary Fig. 5 and Supplementary Note 1). We then estimate the activity ratio $\ln \frac{\gamma_{\mathrm{rel}}^{\text {melt }}, 5}{\gamma_{\mathrm{reO}}^{\text {melt }}}$ by relating it to the interaction parameters between all the components following ref. ${ }^{19}$. Moreover, the experimental results on ferric iron content $\left(\mathrm{Fe}^{3+} / \Sigma \mathrm{Fe}\right)$ at various conditions (listed in Supplementary Table 2) are fit to the Eq. (3) to resolve the interaction parameters (Supplementary Table 3, Supplementary Fig. 6, Supplementary Note 2). We explore four different methods to fit the interaction parameters, but all models yield very similar redox profiles for MOs (Supplementary Fig. 7). We choose the one with smallest reduced chisquare as the best model and our predicted ferric iron contents (shown in Supplementary Fig. 8) are broadly consistent with the observations by both 1-bar experiments ${ }^{18-22}$ and the recent highpressure experiments ${ }^{12,13,23}$ (Supplementary Note 3).

The redox gradients in MOs of Earth, Mars, and the Moon are calculated using Eq. (3) along a cold thermal profile where $2100 \mathrm{~K}$ is assumed to be temperature at the surface (Fig. 2). Similar results are obtained for a hot geotherm with the surface temperature set at $2500 \mathrm{~K}$ (Supplementary Fig. 9).The uncertainties of all the parameters in Eq. (3) are propagated to calculate the oxygen fugacity using LMFIT package ${ }^{29}$. We use $\Delta V$ of $12.5 \mathrm{~mol} \%$ $\mathrm{Fe}$ in silicate melts as a representative value for Earth ${ }^{30}$ and the Moon $^{31}$ and that of $25 \mathrm{~mol} \% \mathrm{Fe}$ for Mars ${ }^{32}$. This assumption is justified for given mantle compositions of these three planetary bodies (Supplementary Table 4) because of relatively small effects of iron content on $\Delta V$ (Fig. 1). We quantify the redox states in terms of oxygen fugacity relative to IW buffers, that is, $\Delta \mathrm{IW}=$ $\log f_{\mathrm{O}_{2}}-\mathrm{IW}$, where the reference IW is taken from ref. ${ }^{33}$. Since the temperatures considered are higher than the temperature at which this IW buffer is calibrated, we extrapolate this buffer equation to high temperatures ${ }^{12,13}$. We also assume that the bases of MOs are at depths of $55 \mathrm{GPa}^{34}, 14 \mathrm{GPa}^{35}$, and $5 \mathrm{GPa}^{36}$, and the corresponding redox states $(\Delta \mathrm{IW})$ are $-2,-1.5$, and -2 for Earth, Mars, and the Moon, respectively ${ }^{13}$. These redox values are representatives for terrestrial bodies when the molten iron ponds are assumed to be in local equilibrium with the overlaying $\mathrm{MOs}^{13}$. The pressures considered here are based on the single stage model and the complete equilibrium between the silicate melt and iron melt. More general consideration of magma ocean depths is discussed below.

Along both thermal profiles considered, the absolute oxygen fugacities of the MOs of Earth, Mars, and the Moon all increase with depth, though more gradually at greater depths (Supplementary Fig. 9). This is expected because $\Delta V$ decreases with increasing pressure and always remains positive over the conditions we investigate. However, the relative oxygen fugacity $(\Delta \mathrm{IW})$ first increases slightly with pressure by $\sim 0.3 \log$ unit in the uppermost mantle and then gradually decreases with pressure throughout the rest of mantle (Fig. 2). Our results show that the 


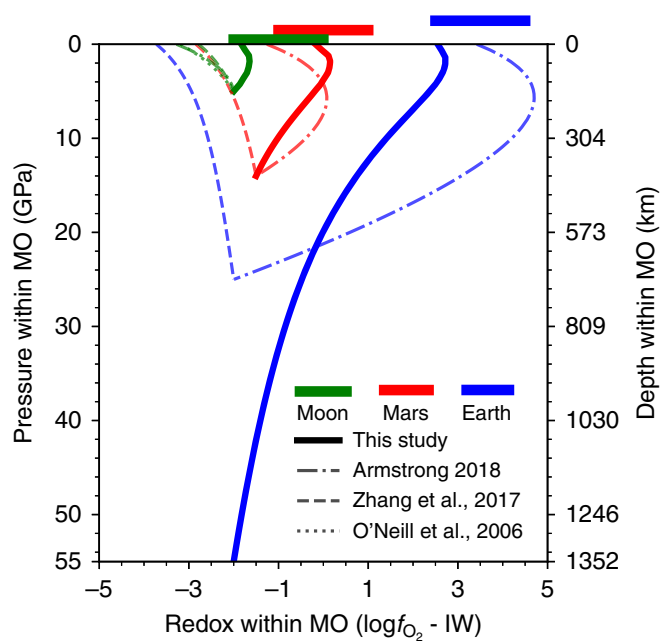

Fig. 2 Redox profiles of magma oceans (MOs) for Earth, Mars, and the Moon. Redox state defined by $\Delta \mathrm{IW}=\log f_{\mathrm{O}_{2}}-\mathrm{IW}$ is shown as a function of pressure along a cold thermal profile.The $\mathrm{MO}$ bases are taken to be at depths corresponding to 55,14 , and $5 \mathrm{GPa}$ with redox states ( $\Delta \mathrm{IW}$ ) of -2 , -1.5 , and -2 for Earth, Mars, and the Moon, respectively. The $1 \sigma$ standard deviation of the oxygen fugacity is $\sim 0.5 \mathrm{log}$ unit for this study (thick solid curves). The previous model results from ref. ${ }^{12}$ (dashed-dotted curves) and ref. ${ }^{23}$ (dotted curves) are also shown within their applicable ranges. For the model of ref. ${ }^{13}$ (dashed curves), we follow the model to extrapolate to 15 and $25 \mathrm{GPa}$ to predict the oxygen fugacities of the Martian and Earth's MOs, respectively. The redox states of the present (upper) mantle of Earth, Mars, and the Moon are displayed in horizontal bars ${ }^{55}$. See Supplementary Fig. 9 for MO redox profiles along a hot thermal profile and Supplementary Fig. 3 for the thermal profiles.

upper mantle should have been relatively more oxidized. Therefore, an oxidized upper mantle is a natural consequence of a MO because the pressure- and temperature-dependent $\Delta V$ and $\Delta G_{r}^{0}\left(P_{0}, T\right)$ of the Eq. (3) make ferric iron increasingly stable at greater depths even at relatively reduced conditions (this raises $\mathrm{Fe}^{3+} / \Sigma \mathrm{Fe}$ of silicate melts in equilibrium with metal alloy). Additionally, our derived redox profiles of Earth, Mars, and the Moon are nearly parallel owing to the similar $\Delta V$ values. They show that Earth is $\sim 2 \log$ units more oxidized than Mars which, in turn, is $\sim 2 \log$ units more oxidized than the Moon at the same depth. This order of relative redox states of the MOs of the early Earth, Mars, and the Moon coincides with that of their presentday mantles, implying a possible inheritance of present-day oxidation states of these planets from their early MOs.

The comparison between the predicted redox profile of the MO with that of the present-day mantle for each planet informs us how the MO stage influences the subsequent redox evolution of each planet throughout its history. The oxidation state of the uppermost mantle of the present-day Earth is near IW $+3.5^{37}$ and has remained constant within $\sim 1.0$ log unit since at least the early Archean ${ }^{6,7}$. Our predicted redox state of the uppermost MO of Earth is at the lower bound of present-day values. Likewise, the ferric iron content corresponding to this redox profile is $1.0-3.5 \%$, overlapping with the lower end of the present-day ferric iron abundances of the upper mantle ${ }^{37}$. The predicted redox state and ferric iron content suggest that Earth's oxidizing uppermost mantle is a natural outcome of the thermodynamic equilibrium across the deep MO during the MO stage. Secondary contributions may arise from other mechanisms, including disproportionation of $\mathrm{Fe}^{2+}$ in the lower mantle by crystallization of bridgmanite $^{38,39}$, and/or late accretion of oxidized materials ${ }^{40,41}$. Compared to the silicate Earth, the Martian uppermost mantle is less oxidized with $f_{\mathrm{O}_{2}} \sim \mathrm{IW}^{11,42}$, which is consistent with our predicted redox state of the shallow Martian MO. This similarity may suggest negligible effects of subsequent tectonic processes and other oxidizing mechanisms mentioned above on the redox state of the Martian mantle ${ }^{43}$. Lunar basalts record oxygen fugacity ranging from IW to IW $-2^{8-10}$ and our predicted redox state falls into the lower end of the observed values. Our predicted redox profiles differ considerably from those based on previous models (Fig. 2). Previous models have generally predicted relatively more reduced MOs of the Moon and Mars and either very reducing ${ }^{13}$ or very oxidizing $\mathrm{MO}$ of Earth ${ }^{12}$. It is important to note that the previously used data are limited with respect to pressure and temperature, for example, up to $3 \mathrm{GPa}$ and 1673 $\mathrm{K}^{23}, 7 \mathrm{GPa}$ and $2023 \mathrm{~K}^{13}$, and $23 \mathrm{GPa}$ and $2300 \mathrm{~K}^{12}$ (Supplementary Table 3 ).

We also investigate the effects of varying depth of the MOs on the redox states of the surface and equilibrium ferric iron content (Fig. 3 and Supplementary Fig. 10). The redox states of the MO bases $(\Delta \mathrm{IW})$ are assumed to be fixed at $-2,-1.5,-2$, respectively, for Earth, Mars, and the Moon. A deeper MO generally shifts upwards its oxygen fugacity profile at shallower depths (Fig. 3a). The redox states and ferric iron contents of the Lunar and Martian MOs are marginally affected due to their small sizes. In contrast, the Earth's MO may have reached 25-90 GPa based on moderately siderophile elements abundances, assuming models for single or multi-stage core formation with partial or complete equilibrium between impactor and proto Earth $^{34,44}$. The oxygen fugacity of Earth's surface would decrease by $\sim 1.5 \log$ units if the base of MO moved upwards from $55 \mathrm{GPa}$ to $25 \mathrm{GPa}$. Concurrently, $\mathrm{Fe}^{3+} / \Sigma \mathrm{Fe}$ would also drop by a factor of two. An even deeper magma ocean may induce the spin transition of iron in the silicate melts. However, the effect of the spin transition on the oxygen fugacity is shown to be insignificant within the MO thermal profiles considered here (Methods and Supplementary Fig. 11). Note our assumption that the ferric iron distribution is homogeneous within the MO due to vigorous convection. However, this ferric iron content profile likely evolves during the solidification of the MO. The evolution is controlled by how the MO crystallizes and the partitioning of iron species between the melt and crystal, which are still poorly constrained. Nevertheless, our study suggests that the whole mantles of Earth and Mars could have been as enriched in ferric iron as the present-day upper mantle since the MO stage.

Chemistry of early atmospheres. The redox states of the MOs may have dictated the chemical speciation of the early atmospheres. For simplicity, we consider a case where the early atmosphere is at chemical equilibrium with the underlying $\mathrm{MO}^{3}$ and use the approach of ref. ${ }^{45}$ to calculate the speciation of volatiles. Based on our results shown in Fig. 3, the redox state at the MO surface corresponds to $\sim \mathrm{IW}+2$ for Earth, $\sim \mathrm{IW}-0.3$ for Mars, and $\sim \mathrm{IW}-2$ for the Moon. Assuming a simple $\mathrm{C}-\mathrm{O}-\mathrm{H}$ atmosphere with a mass $\mathrm{H} / \mathrm{C}$ ratio of 0.5 at 1 bar and $1800 \mathrm{~K}$, we show that the Earth's early atmosphere would be enriched in $\mathrm{H}_{2} \mathrm{O}$ $(\sim 70 \mathrm{~mol} \%)$ and $\mathrm{CO}_{2}(\sim 15 \mathrm{~mol} \%)$ but depleted in $\mathrm{CO}$ and $\mathrm{H}_{2}$. The early Martian atmosphere would consist of $\mathrm{H}_{2} \mathrm{O}$ and $\mathrm{H}_{2}$ in almost equal amounts (each $\sim 40 \mathrm{~mol} \%$ ), $\sim 15 \mathrm{~mol} \% \mathrm{CO}$, and $\sim 5$ mol\% $\mathrm{CO}_{2}$. In contrast, the early lunar atmosphere would be enriched in $\mathrm{H}_{2}(>70 \mathrm{~mol} \%)$ and $\mathrm{CO}(\sim 20 \mathrm{~mol} \%)$ and relatively depleted in $\mathrm{H}_{2} \mathrm{O}(10 \mathrm{~mol} \%)^{3,45}$ (Fig. 4). These early atmospheres further evolve as the planets cool down. The speciation and mass of the atmosphere would likely change over time due to the thermodynamic re-equilibrium, hydrodynamic loss, as well as subsequent degassing and ingassing/regassing. Nevertheless, these distinct early atmospheric compositions may have fundamentally 

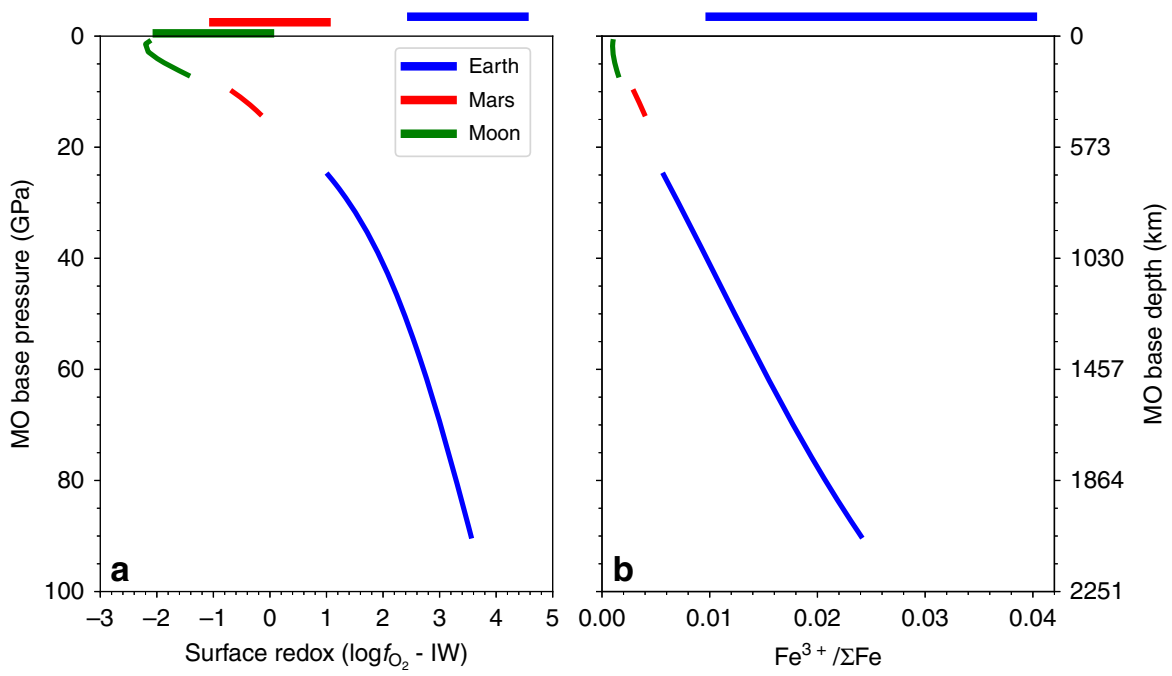

Fig. 3 Redox state and ferric iron contents at the surfaces of magma oceans (MOs) of varying depths. The calculated relative redox state a and $\mathrm{Fe}^{3+} / \Sigma \mathrm{Fe}$ ratio $\mathbf{b}$ of MOs of Earth (blue), Mars (red) and the Moon (green) as a function of pressuresat the base of MOs considering a cold thermal profile (Supplementary Note 2). Calculations are performed at the plausible pressure ranges of the bases of the MOs suggested by previous studies ${ }^{34-36,44}$. The redox state/ferric iron content of the present (upper) mantle of Earth (blue), Mars (red) and the Moon (green) are presented as horizontal bars ${ }^{55}$. The ferric iron contents of the mantle of Mars and the Moon are poorly constrained (not shown) because the available samples suffer from alterations and post-formation oxidations and cannot reflect the ferric iron contents of the source mantle 56,57 . The $1 \sigma$ standard deviation is $\sim 0.5$ log unit for of the oxygen fugacity and $\sim 0.03-0.06$ for the ferric iron content.

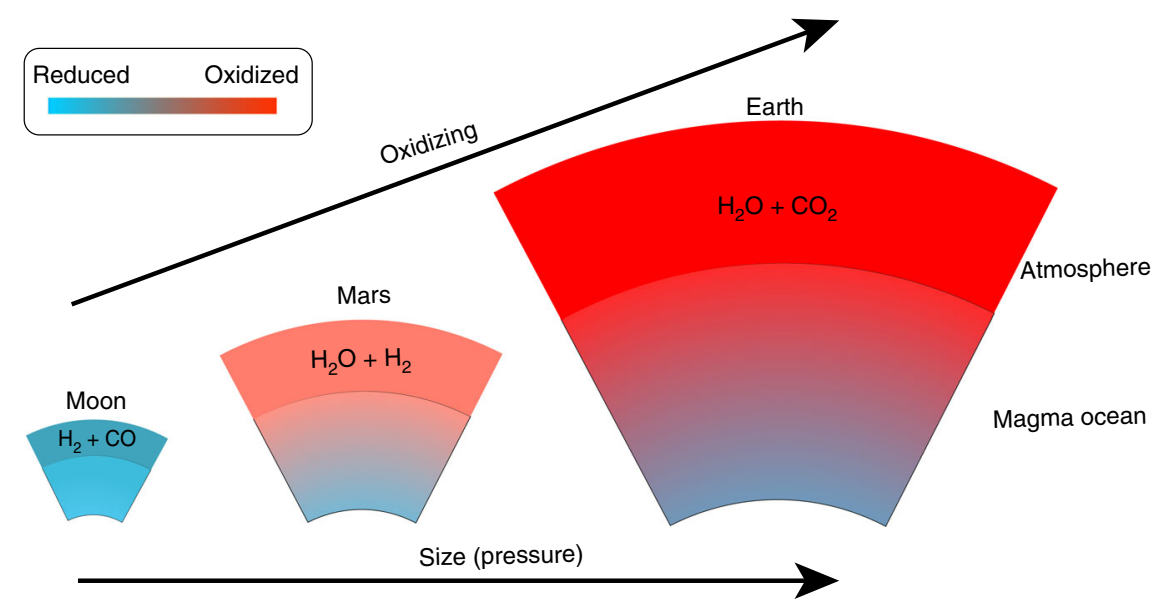

Fig. 4 Inferred compositions of early atmospheres. The redox states of magma oceans and the dominant chemical speciation of the overlying atmospheres are shown for Earth, Mars and the Moon. Refer to the text for estimated fraction of each species. The thicknesses and oxidation states of atmospheres are not scaled.

influenced the subsequent evolution of these terrestrial planets, including climate, magma ocean solidification, and the evolution of surficial conditions 3,46 .

The vertical gradient in the MO redox state predicted here may also apply to other rocky planets where MOs were once formed. For example, Earth's sister planet Venus is of similar size and has similar iron content. The redox state of the post-MO upper mantle of Venus, to first order, may be similar to that of Earth and could be tested by future Venusian missions. In addition, super-Earths close to their host stars may have MOs extended to various depths and their atmospheres can potentially be detected in the near future with space telescope missions ${ }^{47}$.

\section{Methods}

Computational details. First-principles molecular dynamics (FPMD) simulations were carried out using the VASP software ${ }^{48}$ in the NVT-canonical ensemble with temperature controlled by a Nosé thermostat ${ }^{49}$. The projector augmented wave potentials $s^{50,51}$ were employed together with the generalized gradient approximation (GGA) to the exchange-correlation potential ${ }^{52}$. The plane-wave basis cutoff was set at $400 \mathrm{eV}$ and Brillouin zone sampling was performed at the Gamma point. Pulay stress corrections were applied to the calculated pressures. We simulated

$\mathrm{Mg}_{14} \mathrm{Fe}_{2} \mathrm{Si}_{16} \mathrm{O}_{48}$ (ferrous) and $\mathrm{Mg}_{14} \mathrm{Fe}_{2} \mathrm{Si}_{16} \mathrm{O}_{49}$ (ferric) melts for $12.5 \mathrm{~mol} \%$ iron and $\mathrm{Mg}_{12} \mathrm{Fe}_{4} \mathrm{Si}_{16} \mathrm{O}_{48}$ (ferrous) and $\mathrm{Mg}_{12} \mathrm{Fe}_{4} \mathrm{Si}_{16} \mathrm{O}_{50}$ (ferric) melts for $25 \mathrm{~mol} \%$ iron. Iron was set to be in high-spin state in all simulations. At each volume, the system was initially melted and thermalized at $6000 \mathrm{~K}$, and then subsequently quenched down to desired lower temperatures of 4000, 3000, 2500, and $2000 \mathrm{~K}$. Simulations were run for 10-30 picoseconds with time step of 1 femtosecond. Further details can be found in ref. ${ }^{53}$.

Calculation of volume difference $(\Delta \mathbf{V})$. The molar volume difference between $\mathrm{FeO}_{1.5}$ and $\mathrm{FeO}$ defined as $\Delta V=V_{\mathrm{FeO}_{1.5}}-V_{\mathrm{FeO}}$ is calculated as the volume difference between the ferric and ferrous iron-bearing silicate melts. Take the silicate melt with $12.5 \mathrm{~mol} \%$ iron as an example. The volumes of $\mathrm{Mg}_{14} \mathrm{Fe}_{2} \mathrm{Si}_{16} \mathrm{O}_{48}$ and

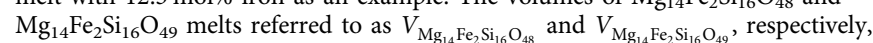
are calculated at the same pressure and temperature conditions using the resolved equation of state parameters (Supplementary Table 1). These volumes can be 
related to the partial volumes of components by:

$$
V_{\mathrm{Mg}_{14} \mathrm{Fe}_{2} \mathrm{Si}_{16} \mathrm{O}_{48}}=14 V_{\mathrm{MgSiO}_{3}}+2 V_{\mathrm{FeO}}+2 V_{\mathrm{SiO}_{2}}+V^{\mathrm{E} \text {,reduced }}
$$

and

$$
V_{\mathrm{Mg}_{14} \mathrm{Fe}_{2} \mathrm{Si}_{16} \mathrm{O}_{49}}=14 V_{\mathrm{MgSiO}_{3}}+2 V_{\mathrm{FeO}_{1.5}}+2 V_{\mathrm{SiO}_{2}}+V^{\mathrm{E}, \text { oxidized }},
$$

where $V^{\mathrm{E} \text {,reduced }}$ and $V^{\mathrm{E}, \text { oxidized }}$ are the excess volumes for reduced and oxidized systems, respectively, and are sensitive to the amount of iron. Several previous lowpressure experiments show that the excess terms are small for silicate melts if $\mathrm{Na}_{2} \mathrm{O}, \mathrm{Al}_{2} \mathrm{O}_{3}$, and $\mathrm{TiO}_{2}$ components are absent ${ }^{18,24}$. In this case, we can approximate $\Delta V$ by

$$
\Delta V=V_{\mathrm{FeO}_{1.5}}-V_{\mathrm{FeO}} \approx\left(V_{\mathrm{Mg}_{14} \mathrm{Fe}_{2} \mathrm{Si}_{16} \mathrm{O}_{49}}-V_{\mathrm{Mg}_{14} \mathrm{Fe}_{2} \mathrm{Si}_{16} \mathrm{O}_{48}}\right) / 2
$$

Similarly, for $25 \mathrm{~mol} \%$ iron content, we use

$$
\Delta V=V_{\mathrm{FeO}_{1.5}}-V_{\mathrm{FeO}} \approx\left(V_{\mathrm{Mg}_{12} \mathrm{Fe}_{4} \mathrm{Si}_{16} \mathrm{O}_{50}}-V_{\mathrm{Mg}_{12} \mathrm{Fe}_{4} \mathrm{Si}_{16} \mathrm{O}_{48}}\right) / 4 .
$$

By using the above equation to calculate $\Delta V$, we assume that $V^{\mathrm{E} \text {,oxidized }}$ and $V^{\mathrm{E} \text {,reduced }}$ take small similar values so $V^{\mathrm{E} \text {,oxidized }}-V^{\mathrm{E} \text {,reduced }} \approx 0$. If $V^{\mathrm{E} \text {,oxidized }}-$ $V^{\mathrm{E}, \text { reduced }}$ is a large non-zero value, one would expect that the $\Delta V$ differs significantly between the two compositions considered ( 12.5 and $25 \mathrm{~mol} \%$ iron in silicate melts). However, our calculated results show that the $\Delta V$ values of 12.5 and $25 \mathrm{~mol} \%$ iron contents differ slightly from each other and the difference diminishes especially at high pressures, which justifies our assumptions.

It should be noted that the small excess volume is not conflicted with the large Margules interaction parameters resolved for silicate melts. The excess volume is thermodynamically defined as $V^{\mathrm{E}}=\left(\frac{\partial G_{\text {mix }}}{\partial \mathrm{P}}\right)_{\mathrm{T}}=\left(\frac{\partial H_{\text {mix }}}{\partial \mathrm{P}}\right)_{\mathrm{T}}$, where $G_{\text {mix }}$ and $H_{\text {mix }}$ are the Gibbs free energy and enthalpy of mixing, respectively; $P$ is pressure; and $T$ is temperature. $H_{\text {mix }}$ is a function of interaction parameter $(W)$ and composition ${ }^{54}$. For a binary system with endmember components $\mathrm{A}$ and $\mathrm{B}, H_{\text {mix }}=W X_{\mathrm{A}} X_{\mathrm{B}}$, where $X_{\mathrm{A}}$ and $X_{\mathrm{B}}$ are the molar fractions of A and B, respectively. Therefore, a small $V^{\mathrm{E}}$ requires that the pressure derivative of the interaction parameter to be small but does not necessarily mean that the value of $W$ is small. Indeed, both in this study and many other studies ${ }^{13,23}, W$ is assumed to be pressure independent, which is in line with the assumption that $V^{\mathrm{E}}$ is small.

Effects of spin transition of iron on $\Delta V$ and oxygen fugacity. Both ferric and ferrous irons undergo electronic spin transitions at high pressure as predicted by a recent FPMD study ${ }^{53}$. The high- to low-spin transition of $\mathrm{Fe}^{3+}$ and $\mathrm{Fe}^{2+}$ occurs gradually over pressure intervals centered around 90 and $110 \mathrm{GPa}$, respectively, at $3000 \mathrm{~K}$. These transition pressures are higher than the maximum pressures of the magma oceans considered in this study (Fig. 2). As both $\mathrm{Fe}^{3+}$ and $\mathrm{Fe}^{2+}$ will be mostly in high-spin (HS) state at relevant magma ocean pressures, we evaluate the volume difference between $\mathrm{FeO}_{1.5}$ and $\mathrm{FeO}$ as $\Delta V=V_{\mathrm{HS}}^{\mathrm{FeO}}{ }_{1.5}-V_{\mathrm{HS}}^{\mathrm{FeO}}$. However, all $\mathrm{Fe}^{3+}$ and $\mathrm{Fe}^{2+}$ will not undergo the HS-LS transition at a given condition. This means that the spin transition-induced changes in volume also contribute to our $\Delta V$ evaluation. We assess the spin effects on $\Delta V$ using the spin phase diagrams from Karki et al. ${ }^{53}$. Considering exact HS and LS distributions for both ferrous and ferric irons, we can evaluate the volume difference between $\mathrm{FeO}_{1.5}$ and $\mathrm{FeO}$ as

$$
\begin{aligned}
\Delta V_{\text {exact }}= & \left(V_{\mathrm{HS}}^{\mathrm{FeO}_{1.5}}-V_{\mathrm{HS}}^{\mathrm{FeO}}\right)-n_{\mathrm{LS}}^{\mathrm{Fe}^{3+}}\left(V_{\mathrm{HS}}^{\mathrm{FeO}_{1.5}}-V_{\mathrm{LS}}^{\mathrm{FeO}_{1.5}}\right) \\
& +n_{\mathrm{LS}}^{\mathrm{Fe}^{2+}}\left(V_{\mathrm{HS}}^{\mathrm{FeO}}-V_{\mathrm{LS}}^{\mathrm{FeO}}\right),
\end{aligned}
$$

where $V_{\mathrm{HS}}^{\mathrm{FeO}}{ }^{1.5}-V_{\mathrm{HS}}^{\mathrm{FeO}}=\Delta V$ has been rigorously constrained in this study. $n_{\mathrm{LS}}^{\mathrm{Fe}^{3+}}$ and $n_{\mathrm{LS}}^{\mathrm{Fe}^{2+}}$ represent the fractions of $\mathrm{Fe}^{3+}$ and $\mathrm{Fe}^{2+}$ in low-spin (LS) state, respectively (satisfying the relations $n_{\mathrm{HS}}^{\mathrm{Fe}^{3+}}+n_{\mathrm{LS}}^{\mathrm{Fe}^{3+}}=n_{\mathrm{HS}}^{\mathrm{Fe}^{2+}}+n_{\mathrm{LS}}^{\mathrm{Fe}^{2+}}=1$, where $n_{\mathrm{HS}}^{\mathrm{Fe}^{3+}}$ and $n_{\mathrm{HS}}^{\mathrm{Fe}^{2+}}$ represent the corresponding HS fractions) and their values as a function of pressure and temperature for silicate melt with $25 \% \mathrm{Fe}$ can be found in ref. ${ }^{53}$. Karki et al. ${ }^{53}$ also evaluated the $V_{\mathrm{HS}}^{\mathrm{FeO}}{ }_{1.5}-V_{\mathrm{LS}}^{\mathrm{FeO} \mathrm{I}_{1.5}}$ and $V_{\mathrm{HS}}^{\mathrm{FeO}}-V_{\mathrm{LS}}^{\mathrm{FeO}}$ to be constant with respect to pressure within the computational uncertainties. At 3000 and 4000 $\mathrm{K}, V_{\mathrm{HS}}^{\mathrm{FeO}} \mathrm{O}_{1.5}-V_{\mathrm{LS}}^{\mathrm{FeO}}{ }_{1.5} \approx 1.25 \mathrm{~cm}^{3} \mathrm{~mol}^{-1}$ and $1.00 \mathrm{~cm}^{3} \mathrm{~mol}^{-1}$, respectively, and $V_{\mathrm{HS}}^{\mathrm{FeO}}-$ $V_{\mathrm{IS}}^{\mathrm{FeO}} \approx 1.75 \mathrm{~cm}^{3} \mathrm{~mol}^{-1}$ and $1.10 \mathrm{~cm}^{3} \mathrm{~mol}^{-1}$, respectively ${ }^{53}$. We calculate the difference of $\Delta V_{\text {exact }}$ and $\Delta V$ at 3000 and $4000 \mathrm{~K}$ as well as the difference of the oxygen fugacity using these two volume differences (Supplementary Fig. 11)

At pressures less than $60 \mathrm{GPa}$, we find that the deviation of the volume difference caused by considering the spin transition is less than $3 \%$, so the oxygen fugacity does not change much when spin effects are included (Supplementary Fig. 11). With increasing pressure, the magnitude of $\left(\Delta V_{\text {exact }}-\Delta V\right)$ further increases and bounces back at around $100 \mathrm{GPa}$, at which the fraction of low-spin $\mathrm{Fe}^{3+}$ reaches around $50 \%$. Note that at pressures greater than $80 \mathrm{GPa}$, the temperature of the $\mathrm{MO}$ is around $3500 \mathrm{~K}$ for a cold thermal profile and continues increasing with pressure. Therefore, the results at $4000 \mathrm{~K}$ are more relevant at these pressures. Overall, neglecting the spin transition of Fe tends to overestimate the oxygen fugacity, especially at high pressures. The maximum deviation occurs around $120 \mathrm{GPa}$, which is $\sim 0.6 \log$ units, comparable to the uncertainties of our model prediction $(\sim 0.5)$. Therefore, we consider the effects of spin transition of iron on the redox state of MOs to be mostly insignificant.

\section{Data availability}

Authors can confirm that all relevant data are included in the paper and/or its supplementary information files.

Received: 20 September 2019; Accepted: 20 March 2020; Published online: 24 April 2020

\section{References}

1. Armstrong, L. S., Hirschmann, M. M., Stanley, B. D., Falksen, E. G. \& Jacobsen, S. D. Speciation and solubility of reduced $\mathrm{C}-\mathrm{O}-\mathrm{H}-\mathrm{N}$ volatiles in mafic melt: Implications for volcanism, atmospheric evolution, and deep volatile cycles in the terrestrial planets. Geochim. Cosmochim. Acta 171, 283-302 (2015).

2. Stanley, B. D., Hirschmann, M. M. \& Withers, A. C. Solubility of $\mathrm{COH}$ volatiles in graphite-saturated martian basalts. Geochim. Cosmochim. Acta 129, 54-76 (2014)

3. Hirschmann, M. M. Magma ocean influence on early atmosphere mass and composition. Earth Planet. Sci. Lett. 341-344, 48-57 (2012).

4. Wetzel, D. T., Rutherford, M. J., Jacobsen, S. D., Hauri, E. H. \& Saal, A. E. Degassing of reduced carbon from planetary basalts. PNAS. 110, 8010-8013 (2013).

5. Dingwell, D. B. Redox viscometry of some Fe-bearing silicate melts. Am Mineralogist 76, 1560-1562 (1991).

6. Li, Z.-X. A. \& Lee, C.-T. A. The constancy of upper mantle $\mathrm{fO}_{2}$ through time inferred from V/Sc ratios in basalts. Earth Planet. Sci. Lett. 228, 483-493 (2004).

7. Trail, D., Watson, E. B. \& Tailby, N. D. The oxidation state of Hadean magmas and implications for early Earth's atmosphere. Nature 480, 79 (2011)

8. Wadhwa, M. Redox conditions on small bodies, the Moon and Mars. Rev. Mineral. Geochem. 68, 493-510 (2008).

9. Weitz, C. M., Rutherford, M. J. \& Head, J. W. Oxidation states and ascent history of the Apollo 17 volcanic beads as inferred from metal-glass equilibria. Geochim. Cosmochim. Acta 61, 2765-2775 (1997).

10. Nicholis, M. G. \& Rutherford, M. J. Graphite oxidation in the Apollo 17 orange glass magma: implications for the generation of a lunar volcanic gas phase. Geochim. Cosmochim. Acta 73, 5905-5917 (2009).

11. Herd, C. D. K., Papike, J. J. \& Brearley, A. J. Oxygen fugacity of martian basalt from electron microprobe oxygen and TEM-EELS analyses of Fe-Ti oxides. Am. Mineralogist 86, 1015-1024 (2001).

12. Armstrong, K., Frost, D. J., McCammon, C. A., Rubie, D. C. \& Boffa Ballaran T. Deep magma ocean formation set the oxidation state of Earth's mantle. Science 365, 903-906 (2019)

13. Zhang, H. L., Hirschmann, M. M., Cottrell, E. \& Withers, A. C. Effect of pressure on $\mathrm{Fe}^{3+} / \Sigma \mathrm{Fe}$ ratio in a mafic magma and consequences for magma ocean redox gradients. Geochim. Cosmochim. Acta 204, 83-103 (2017).

14. Schaefer, L. \& Elkins-Tanton, L. T. Magma oceans as a critical stage in the tectonic development of rocky planets. Philos T R Soc. A 376, 20180109 https://doi.org/10.1098/rsta.2018.0109 (2018).

15. Stevenson, D. J. Models of the Earths core. Science 214, 611-619 (1981)

16. Rubie, D. C., Nimmo, F.\& Melosh, H. J. in Treatise on Geophysics (2nd Edition) (ed. Gerald Schubert) 43-79 (Elsevier, 2015).

17. Righter, K. \& Ghiorso, M. S. Redox systematics of a magma ocean with variable pressure-temperature gradients and composition. PNAS. 109, 11955-11960 (2012).

18. Kress, V. C. \& Carmichael, I. S. E. The compressibility of silicate liquids containing $\mathrm{Fe}_{2} \mathrm{O}_{3}$ and the effect of composition, temperature, oxygen fugacity and pressure on their redox states. Contributions Mineral. Petrol. 108, 82-92 (1991).

19. Jayasuriya, K. D., Campbell, S. J., Berry, A. J. \& O’Neill, H. S. C. A Mössbauer study of the oxidation state of Fe in silicate melts. Am. Mineralogist 89, 1597-1609 (2004)

20. Thornber, C. R., Roeder, P. L. \& Foster, J. R. The effect of composition on the ferric-ferrous ratio in basaltic liquids at atmospheric pressure. Geochim. Cosmochim. Acta 44, 525-532 (1980).

21. Sack, R. O., Carmichael, I. S. E., Rivers, M. \& Ghiorso, M. S. Ferric-ferrous equilibria in natural silicate liquids at 1 bar. Contributions Mineral. Petrol. 75, 369-376 (1981).

22. Kress, V. C. \& Carmichael, I. S. E. Stoichiometry of the iron oxidation reaction in silicate melts. Am. Mineralogist 73, 1267-1274 (1988).

23. O’Neill, H. S. C. et al. An experimental determination of the effect of pressure on the $\mathrm{Fe}^{3+} / \Sigma \mathrm{Fe}$ ratio of an anhydrous silicate melt to $3.0 \mathrm{GPa}$. Am. Mineralogist 91, 404-412 (2006).

24. Lange, R. A. \& Carmichael, I. S. E. Densities of $\mathrm{Na}_{2} \mathrm{O}-\mathrm{K}_{2} \mathrm{O}-\mathrm{CaO}-\mathrm{MgO}-\mathrm{FeO}-$ $\mathrm{Fe}_{2} \mathrm{O}_{3}-\mathrm{Al}_{2} \mathrm{O}_{3}-\mathrm{TiO}_{2}-\mathrm{SiO}_{2}$ liquids: new measurements and derived partial molar properties. Geochim. Cosmochim. Acta 51, 2931-2946 (1987). 
25. Rigden, S. M., Ahrens, T. J. \& Stolper, E. M. High-pressure equation of state of molten anorthite and diopside. J. Geophys. Res. Solid Earth 94, 9508-9522 (1989).

26. Miller, G. H., Stolper, E. M. \& Ahrens, T. J. The equation of state of a molten komatiite: 1 Shock wave compression to $36 \mathrm{GPa}$. J. Geophys. Res. Solid Earth 96, 11831-11848 (1991).

27. Komabayashi, T. \& Fei, Y. Internally consistent thermodynamic database for iron to the Earth's core conditions. J. Geophys. Res. Solid Earth 115, B03202 (2010).

28. Frost, D. J. et al. Partitioning of oxygen between the Earth's mantle and core. J. Geophys. Res. Solid Earth 115, B02202 (2010).

29. Newville, M., Stensitzki, T., Allen, D. B. \& Ingargiola, A. LMFIT: Non-Linear Least-Square Minimization and Curve-Fitting for Python (Zenodo, 2014) https://doi.org/10.5281/zenodo.11813.

30. McDonough, W. F. \& Sun, S. S. The composition of the Earth. Chem. Geol. 120, 223-253 (1995).

31. Elardo, S. M., Draper, D. S. \& Shearer, C. K. Lunar Magma Ocean crystallization revisited: Bulk composition, early cumulate mineralogy, and the source regions of the highlands Mg-suite. Geochim. Cosmochim. Acta 75, 3024-3045 (2011).

32. Bertka, C. M. \& Fei, Y. Mineralogy of the Martian interior upto core-mantle boundary pressure. J. Geophys. Res. Solid Earth. 102, 5251-5264 (1997).

33. Campbell, A. J. et al. High pressure effects on the iron-iron oxide and nickelnickel oxide oxygen fugacity buffers. Earth Planet. Sci. Lett. 286, 556-564 (2009).

34. Fischer, R. A. et al. High pressure metal-silicate partitioning of $\mathrm{Ni}, \mathrm{Co}, \mathrm{V}, \mathrm{Cr}$, $\mathrm{Si}$, and O. Geochim. Cosmochim. Acta 167, 177-194, https://doi.org/10.1016/j. gca.2015.06.026 (2015)

35. Yang, S. et al. Siderophile and chalcophile element abundances in shergottites: implication for Martian core formation. Meteorit. Planet Sci. 50, 691-714 (2015).

36. Garcia, R. F., Gagnepain-Beyneix, J., Chevrot, S. \& Lognonne, P. Physics of the Earth and Planetary Interiors vol 188, 96-113 (Elsevier, 2012).

37. Frost, D. J. \& McCammon, C. A. The redox state of Earth's mantle. Annu Rev. Earth Pl Sc. 36, 389-420 (2008).

38. Wade, J. \& Wood, B. J. Core formation and the oxidation state of the Earth. Earth Planet. Sci. Lett. 236, 78-95 (2005).

39. Frost, D. J. et al. Experimental evidence for the existence of iron-rich metal in the Earth's lower mantle. Nature 428, 409-412 (2004).

40. O'Neill, H. S. C. The origin of the moon and the early history of the earth-a chemical model. Part 2: the Earth. Geochim. Cosmochim. Acta 55, 1159-1172 (1991).

41. Wood, B. J., Walter, M. J. \& Wade, J. Accretion of the Earth and segregation of its core. Nature 441, 825 (2006).

42. Herd, C. D. K., Borg, L. E., Jones, J. H. \& Papike, J. J. Oxygen fugacity and geochemical variations in the martian basalts: implications for martian basalt petrogenesis and the oxidation state of the upper mantle of Mars. Geochim. Cosmochim. Acta 66, 2025-2036 (2002).

43. Schmidt, M. E., Schrader, C. M. \& McCoy, T. J. The primary $f \mathrm{O}_{2}$ of basalts examined by the Spirit rover in Gusev Crater, Mars: Evidence for multiple redox states in the martian interior. Earth Planet. Sci. Lett. 384, 198-208 (2013).

44. Li, J. \& Agee, C. B. Geochemistry of mantle-core differentiation at high pressure. Nature 381, 686-689 (1996).

45. Kress, V. C., Ghiorso, M. S. \& Lastuka, C. Microsoft EXCEL spreadsheet-based program for calculating equilibrium gas speciation in the $\mathrm{C}-\mathrm{O}-\mathrm{H}-\mathrm{S}-\mathrm{Cl}-\mathrm{F}$ system. Comput. Geosci. 30, 211-214 (2004).

46. Ramirez, R. M. et al. Warming early Mars with $\mathrm{CO}_{2}$ and $\mathrm{H}_{2}$. Nat. Geosci. 7, 59 (2013).

47. Seager, S. \& Deming, D. Exoplanet atmospheres. Annu. Rev. Astron. Astrophys. 48, 631-672 (2010).

48. Kresse, G. \& Furthmüller, J. Efficiency of ab-initio total energy calculations for metals and semiconductors using a plane-wave basis set. Comput. Mater. Sci. 6, 15-50 (1996).

49. Nosé, S. A unified formulation of the constant temperature molecular dynamics methods. J. Chem. Phys. 81, 511-519 (1984).

50. Kresse, G. \& Joubert, D. From ultrasoft pseudopotentials to the projector augmented-wave method. Phys. Rev. B 59, 1758-1775 (1999).

51. Blöchl, P. E., Jepsen, O. \& Andersen, O. K. Improved tetrahedron method for Brillouin-zone integrations. Phys. Rev. B 49, 16223-16233 (1994).
52. Perdew, J. P., Burke, K. \& Ernzerhof, M. Generalized gradient approximation made simple. Phys. Rev. Lett. 77, 3865-3868 (1996)

53. Karki, B. B., Ghosh, D. B., Maharjan, C., Karato, S.-i \& Park, J. Densitypressure profiles of Fe-bearing $\mathrm{MgSiO}_{3}$ liquid: effects of valence and spin states, and implications for the chemical evolution of the lower mantle. Geophys. Res. Lett. 45, 3959-3966 (2018).

54. Deng, J. \& Lee, K. K. M. Melting temperature depression due to the electronic spin transition of iron. Am. Mineralogist 104, 1189-1196 (2019).

55. Cartier, C. \& Wood, B. J. The role of reducing conditions in building mercury. Elements 15, 39-45 (2019).

56. Herd, C. D. K. The oxygen fugacity of olivine-phyric martian basalts and the components within the mantle and crust of Mars. Meteorit. Planet Sci. 38, 1793-1805 (2003)

57. Dyar, M. D. Ferric iron in SNC meteorites as determined by Mössbauer spectroscopy: implications for martian landers and martian oxygen fugacity. Meteorit. Planet Sci. 38, 1733-1752 (2003).

\section{Acknowledgements}

The research is supported by NSF grants (EAR-1321956 and EAR-1551348 to K.K.M.L. and EAR 1764140 to B.B.K.) and from Chinese Academy of Sciences (No. 29 Y93301701 and 51Y8340107 to Z.D.). We benefited from discussions with Colin Jackson and Lars Stixrude. The computing resources were provided by the Yale Center for Research Computing (thanking Kaylea Nelson for guidance) and Louisiana State University High Performance Computing. K.K.M.L.'s effort was partially supported under the auspices of the U.S. Department of Energy by Lawrence Livermore National Laboratory under Contract DE-AC52-07NA27344.

\section{Author contributions}

J.D. and Z.D. conceived and designed the project. J.D. performed the simulations and thermodynamic modelling. J.D., Z.D., B.B.K., D.B.G., and K.K.M.L. contributed to the analysis and manuscript preparation.

\section{Competing interests}

The authors declare no competing interests.

\section{Additional information}

Supplementary information is available for this paper at https://doi.org/10.1038/s41467 020-15757-0.

Correspondence and requests for materials should be addressed to J.D. or Z.D.

Peer review information Nature Communications thanks Jonathan Tucker, Marc Hirschmann and the other anonymous reviewer(s) for their contribution to the peer review of this work. Peer reviewer reports are available.

Reprints and permission information is available at http://www.nature.com/reprints

Publisher's note Springer Nature remains neutral with regard to jurisdictional claims in published maps and institutional affiliations.

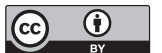

Open Access This article is licensed under a Creative Commons Attribution 4.0 International License, which permits use, sharing, adaptation, distribution and reproduction in any medium or format, as long as you give appropriate credit to the original author(s) and the source, provide a link to the Creative Commons license, and indicate if changes were made. The images or other third party material in this article are included in the article's Creative Commons license, unless indicated otherwise in a credit line to the material. If material is not included in the article's Creative Commons license and your intended use is not permitted by statutory regulation or exceeds the permitted use, you will need to obtain permission directly from the copyright holder. To view a copy of this license, visit http://creativecommons.org/ licenses/by/4.0/.

(c) The Author(s) 2020 